\title{
Comparison of the efficacies of first- generation epidermal growth factor receptor tyrosine kinase inhibitors for brain metastasis in patients with advanced non- small-cell lung cancer harboring EGFR mutations
}

Naoto Aiko ${ }^{1 *}$ (D), Tsuneo Shimokawa ${ }^{1}$, Kazuhito Miyazaki ${ }^{1}$, Yuki Misumi ${ }^{1}$, Yoko Agemi ${ }^{1}$, Mari Ishii ${ }^{2}$, Yukiko Nakamura', Takeharu Yamanaka ${ }^{3}$ and Hiroaki Okamoto ${ }^{1}$

\begin{abstract}
Background: Compared with standard chemotherapy, epidermal growth factor receptor tyrosine kinase inhibitors (EGFR-TKIs) are more effective in patients with advanced non-small-cell lung cancer (NSCLC) harboring EGFR mutations. However, data comparing the efficacies of different EGFR-TKIs, especially regarding the presence of brain metastasis, are lacking.

Methods: EGFR-TKI naive patients with recurrent or stage IIIB/IV NSCLC harboring EGFR mutations, excluding resistance mutations, were enrolled in this study. We retrospectively determined progression-free survival (PFS) using the Kaplan-Meier method with log-rank test in patients treated with either gefitinib or erlotinib, cumulative incidence of central nervous system (CNS) progression using the Fine and Gray competing risk regression model, and favorable prognostic factors for CNS progression by multivariate analysis.

Results: Seventy-seven EGFR-TKI-naive patients were started on either gefitinib $(n=55)$ or erlotinib $(n=22)$ in our hospital from April 2010 to April 2016. Among the patients with brain metastasis, PFS tended to be longer in the erlotinib than in the gefitinib group. In the analysis of cumulative incidence, the probability of CNS progression was lower in the erlotinib group than in the gefitinib group. Particularly, in a subgroup analysis of the patients with brain metastasis, there was a significant difference between the erlotinib and gefitinib groups (hazard ratio 0.25; $95 \%$ confidence interval, $0.08-0.81 ; p=0.021$ ). Of the prognostic factors for CNS progression evaluated, the absence of brain metastasis before EGFR-TKI therapy and receiving erlotinib (vs gefitinib) had a significantly favorable effect on patient prognosis.
\end{abstract}

Conclusion: Although this was a retrospective analysis involving a small sample size, erlotinib is potentially more promising than gefitinib for treatment of brain metastasis in patients with EGFR-mutant NSCLC.

Keywords: Brain metastasis, EGFR TKI, Erlotinib, Gefitinib, Lung cancer, NSCLC

\footnotetext{
* Correspondence: na1435john@gmail.com

${ }^{1}$ Department of Respiratory Medicine, Yokohama Municipal Citizen's Hospital,

56 Okazawa-cho, Hodogaya-ku, Yokohama-city, Kanagawa 240-8555, Japan

Full list of author information is available at the end of the article
}

(c) The Author(s). 2018 Open Access This article is distributed under the terms of the Creative Commons Attribution 4.0 International License (http://creativecommons.org/licenses/by/4.0/), which permits unrestricted use, distribution, and reproduction in any medium, provided you give appropriate credit to the original author(s) and the source, provide a link to the Creative Commons license, and indicate if changes were made. The Creative Commons Public Domain Dedication waiver (http://creativecommons.org/publicdomain/zero/1.0/) applies to the data made available in this article, unless otherwise stated. 


\section{Background}

Approximately $40 \%$ of patients with non-small-cell lung cancer (NSCLC) develop brain metastasis during the course of their disease [1]. And the risk of brain metastasis is greater in patients harboring epidermal growth factor receptor (EGFR) mutations [2].

Compared with standard chemotherapy, EGFR tyrosine kinase inhibitors (EGFR-TKIs) are more effective in patients with advanced NSCLC harboring EGFR mutations [3-6]. Several case reports and studies involving small patient series indicated successful treatment of brain metastasis using EGFR-TKIs [7-10]. However, few studies have compared individual EGFE-TKIs in terms of their efficacy against brain metastasis.

The aim of our study was to evaluate retrospectively the effect of two first-generation EGFR-TKIs (gefitinib and erlotinib) on brain metastasis in patients with NSCLC harboring EGFR mutations.

\section{Methods}

\section{Patient selection}

Patients were chosen from the medical records of Yokohama Municipal Citizen's Hospital if they were recurrent or stage IIIB/IV NSCLC harboring EGFR mutations excluding resistance mutations and received either gefitinib or erlotinib based on physicians' choice for the first EGFR-TKI treatment. The other criteria included Eastern Cooperative Oncology Group performance status (ECOG-PS) ranging from 0 to 3, presence of measurable disease, and adequate organ functions. The exclusion criteria were active infection, uncontrolled angina, myocardial infarction in the previous 6 months, uncontrolled hypertension and diabetes mellitus, interstitial pneumonitis and lung fibrosis as identified on a chest $\mathrm{x}$ - ray, severe mental disorders, and pregnant or lactating women. For assessment disease stage, all patients underwent computed tomography $(\mathrm{CT})$ of the thorax and upper abdomen, either CT or magnetic resonance imaging (MRI) of the brain, and either radioisotopic bone scan or positron emission tomography (PET). CT was basically repeated every 6-8 week to evaluate the target lesions. Tumor response was assessed using the Response Evaluation Criteria in Solid Tumors version 1.1. The study population was assessed using the tumor, node, metastasis staging system (seventh edition of the American Joint Committee on Cancer staging manual).

\section{Statistical analyses}

Progression free survival (PFS) was defined as the interval from the start of EGFR-TKI treatment to disease progression or death from any cause. Alive without progression (data cutoff date, October 31, 2016) and loss to follow-up were censored. PFS was analyzed using the Kaplan-Meier method and compared using the log-rank test. The Fine and Gray competing risk regression model was used to compare cumulative incidence of central nervous system (CNS) progression between gefitinib and erlotinib. Death without CNS progression was considered a competing risk in the analysis, and alive without CNS progression (data cutoff date, October 31, 2016) and loss to follow-up were censored. CNS progression was confirmed by brain MRI or contrast-enhanced CT. In the subgroup-analysis, we analyzed the PFS and cumulative incidence of CNS progression in patients who had brain metastasis before EGFR-TKI administration and those who did not.

The prognostic factors for CNS progression evaluated were age at initiation of EGFR-TKI administration, sex, ECOG PS, presence or absence of brain metastasis before starting EGFR-TKI treatment, and type of EGFR-TKI (gefitinib or erlotinib). Multivariate analysis of the favorable prognostic factors of CNS progression was conducted using the Cox proportional hazards model.

A $P$-value $<0.05$ was considered to indicate a statistically significant difference. All analyses were performed using STATA 14.

\section{Results \\ Patient characteristics}

The patient characteristics are shown in Table 1. In total, 77 patients with NSCLC harboring EGFR mutations were enrolled in this study. Of these, 55 and 22 patients received gefitinib and erlotinib, respectively, as first EGFR-TKI treatment. More patients had poor ECOG PS $(\geqq 2)$ in the gefitinib group (16 [29\%]) compared with erlotinib group (4 [18\%]). Gefitinib (44 [80\%]) was administered as first-line therapy more frequently than erlotinib (9 [41\%]). As for brain metastasis, more of patients who treated with erlotinib have had brain metastasis $(12$ [55\%]) and received radiation therapy (6 [27\%]) prior to EGFR-TKI treatment compared with those treated with gefitinib. No patient who received surgery for brain metastasis and immune check point inhibitor therapy prior to EGFR-TKI was included in both group.

\section{Progression free survival}

Kaplan-Meier plots for PFS are shown in Fig. 1. The median PFS of patients in the erlotinib and gefitinib groups were 11.1 and 9.6 months, respectively $(p=0.860$, Fig. 1a). Among patients with brain metastasis before EGFR-TKI administration, the median PFS of patients in the erlotinib and gefitinib groups were 11.5 and 9.7 months, respectively ( $p=0.257$, Fig. $1 \mathrm{~b})$. Among the patients without brain metastasis, the median PFS of patients in the erlotinib and gefitinib groups were 8.5 and 
Table 1 Patient characteristics

\begin{tabular}{|c|c|c|}
\hline & Gefitinib $(n=55)$ & $\begin{array}{l}\text { Erlotinib } \\
(n=22)\end{array}$ \\
\hline \multicolumn{3}{|l|}{ Sex, n (\%) } \\
\hline Male & $19(35)$ & $11(50)$ \\
\hline Female & $36(65)$ & $11(50)$ \\
\hline Median age, years (range) & $71(46-91)$ & $71(47-83)$ \\
\hline \multicolumn{3}{|l|}{ ECOG PS, n (\%) } \\
\hline $0-1$ & $39(71)$ & $18(82)$ \\
\hline$\geqq 2$ & $16(29)$ & $4(18)$ \\
\hline \multicolumn{3}{|l|}{ TNM stage, $\mathrm{n}(\%)$} \\
\hline $3 B$ & $3(5)$ & $1(5)$ \\
\hline 4 & $43(78)$ & $16(73)$ \\
\hline Recurrence & $9(16)$ & $5(23)$ \\
\hline \multicolumn{3}{|l|}{ Previous chemotherapy regimen, $\mathrm{n}(\%)$} \\
\hline 0 & $44(80)$ & $9(41)$ \\
\hline 1 & $9(16)$ & $7(32)$ \\
\hline$\geqq 2$ & $2(4)$ & $6(27)$ \\
\hline \multicolumn{3}{|l|}{ Brain metastasis, n (\%) } \\
\hline 0 & $40(73)$ & $10(45)$ \\
\hline$\geqq 1$ & $15(27)$ & $12(55)$ \\
\hline $\begin{array}{l}\text { Radiotherapy for brain metastasis before } \\
\text { EGFR-TKI treatment, } \mathrm{n}(\%)\end{array}$ & $4(7)$ & $6(27)$ \\
\hline WBRT & $1(2)$ & $1(4)$ \\
\hline SRT & $3(5)$ & $5(23)$ \\
\hline \multicolumn{3}{|l|}{ EGFR mutation, $\mathrm{n}(\%)$} \\
\hline Exon19 del & $24(44)$ & $6(27)$ \\
\hline Exon21 L858R & $29(53)$ & $15(68)$ \\
\hline Minor & $2(4)$ & $1(5)$ \\
\hline $\begin{array}{l}\text { Dose reduction or intermittent } \\
\text { administration, } \mathrm{n}(\%)\end{array}$ & $13(24)$ & $10(45)$ \\
\hline \multicolumn{3}{|l|}{ Best response, $\mathrm{n}(\%)$} \\
\hline$C R$ & $0(0)$ & $0(0)$ \\
\hline PR & $24(44)$ & $11(50)$ \\
\hline SD & $20(36)$ & $9(41)$ \\
\hline PD & $4(7)$ & $1(5)$ \\
\hline Unknown & $7(13)$ & $1(5)$ \\
\hline \multicolumn{3}{|l|}{ The reason of EGFR-TKI discontinuation, $\mathrm{n}(\%)$} \\
\hline Disease progression & $36(65)$ & $17(77)$ \\
\hline CNS progression & $10(18)$ & $2(9)$ \\
\hline Adverse event & $5(9)$ & $2(9)$ \\
\hline Other & $7(13)$ & $1(5)$ \\
\hline Ongoing & $7(13)$ & $2(9)$ \\
\hline
\end{tabular}

ECOG PS Eastern Cooperative Oncology Group performance status, WBRT whole brain radiotherapy, SRT stereotactic radiotherapy, $C R$ complete response, $P R$ partial response, $S D$ stable disease, $P D$ progressive disease, $C N S$ central nervous system, EGFR-TKI epidermal growth factor receptor tyrosine kinase inhibitor
9.6 months, respectively $(P=0.466$, Fig. $1 \mathrm{c})$. While there was no significant difference in PFS between groups in either subset analysis, there was a tendency for a longer PFS in the erlotinib group than in the gefitinib group among the patients with brain metastasis.

\section{Cumulative incidence of CNS progression}

The cumulative incidence curves are shown in Fig. 2. The cumulative risks of CNS progression at 20 and 40 months were $18 \%$ and $34 \%$, respectively, in the gefitinib group and $12 \%$ and $23 \%$, respectively, in the erlotinib group. The hazard ratio (HR) for the erlotinib group was 0.47 (95\% confidence interval $[\mathrm{CI}], 0.18-1.23 ; p=0.124)$. The subgroup analysis showed a significant difference between the erlotinib and gefitinib group among the patients with brain metastasis before EGFR-TKI administration (HR 0.25; 95\% CI, $0.08-0.81 ; p=0.021$ ), while there was no significant difference (HR 0.57; 95\% CI, 0.13-3.01; $p=0.637$ ) among the patients without brain metastasis.

\section{Favorable prognostic factors of CNS progression}

In the multivariate analysis, the absence of brain metastasis before EGFR-TKI therapy and receiving erlotinib (vs gefitinib) had a significantly favorable effect on CNS progression, while sex, age and ECOG PS had no significant influence. More details are presented in Table 2.

\section{Discussion}

Several retrospective subset studies indicated that gefitinib was more likely to progress brain metastases in EGFR-mutant advanced NSCLC patients than erlotinib. Omuro et al. reported that $33 \%$ of patients treated with gefitinib showed CNS progression as the initial site of progression [11], and Yamamoto et al. reported 3.9\% of patients treated with erlotinib showed CNS progression [12]. However, no prospective studies comparing gefitinib with erlotinib has been reported with regard to CNS progression.

In the PFS analysis of our study for patients with brain metastasis, there was a tendency toward a longer PFS in the erlotinib than in the gefitinib group (Fig. 1b). In the cumulative incidence analysis, the probability of CNS progression was lower in the erlotinib group than in the gefitinib group. Particularly, among the patients who had brain metastasis before EGFR-TKI administration, there was a significant difference between the erlotinib and gefitinib groups (Fig. 2b). In the multivariate analysis, we found that receiving erlotinib (vs gefitinib) and absence of CNS metastasis before EGFR-TKI administration are favorable prognostic factor for CNS progression, while sex, age, and ECOG PS had no significant influence on CNS prognosis.

In a randomized phase 3 trial comparing gefitinib and erlotinib efficacy in lung adenocarcinoma patients 


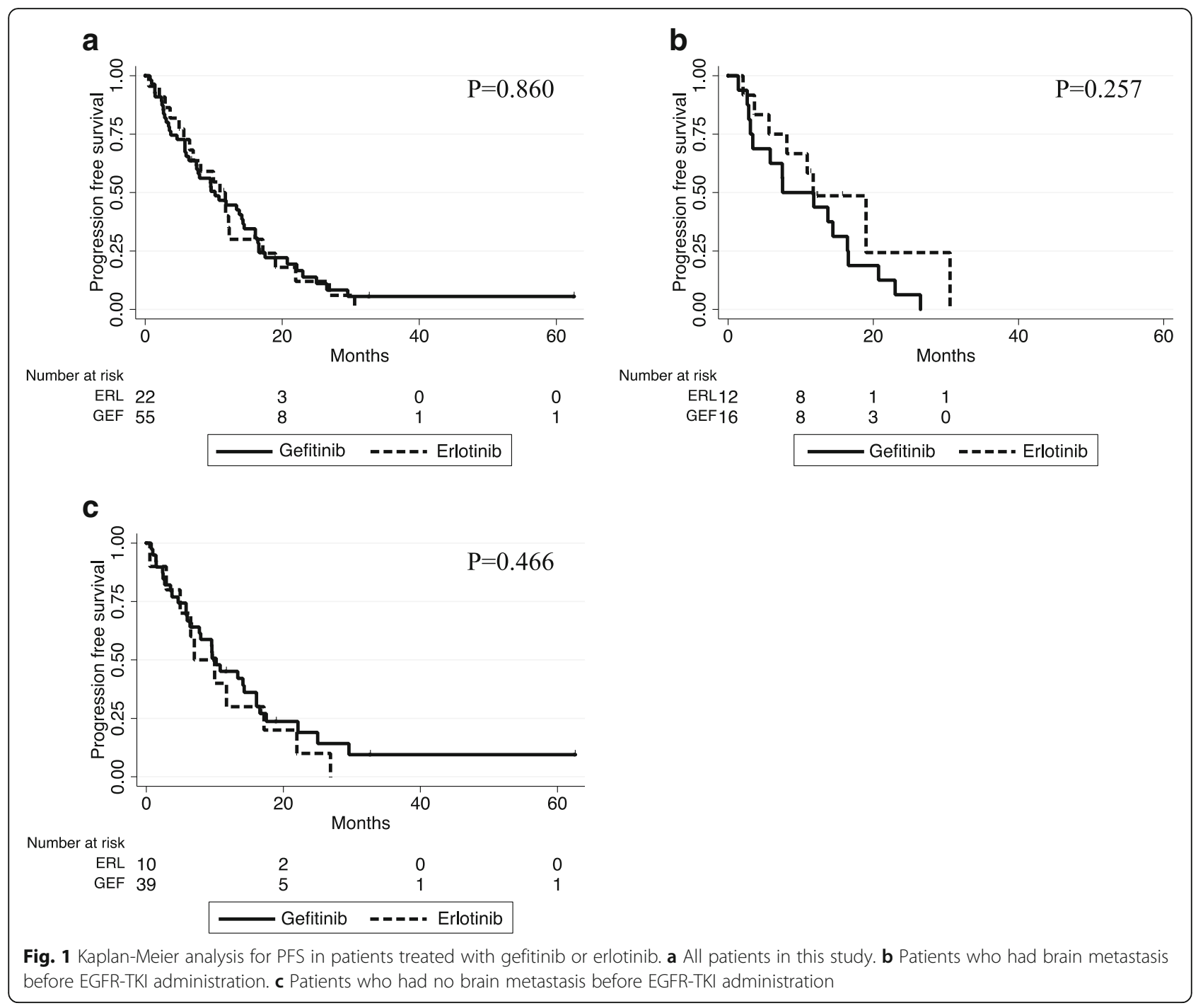

pretreated with chemotherapy, Urata et al. reported equivalent PFS, overall survival (OS), response rate (RR), and disease control rate (DCR) between gefitinib and erlotinib treatments (8.3 and 10.0 months [HR, 1.093; 95\%CI, 0.879 to $1.358 ; p=0.424$ ], 26.5 and 31.4 months [HR, 1.189; 95\%CI, 0.900 to $1.570 ; p=0.221], 58.9 \%$ and $55.0 \%$ [ $p=0.476]$, and $81.7 \%$ and $84.4 \%$ [ $p=0.517]$, respectively) [13]. The results of our study suggested that erlotinib has better efficacy to control CNS metastasis, and contributes to longer PFS among patients with brain metastasis than gefitinib. The maximum blood concentration and area under the curve were $2120 \mathrm{ng} / \mathrm{ml}$ and $38,420 \mathrm{ng} / \mathrm{h} / \mathrm{ml}$ for an erlotinib dose of $150 \mathrm{mg}$ daily (approved dose in Japan) [14] and $307 \mathrm{ng} / \mathrm{ml}$ and $5041 \mathrm{ng} / \mathrm{h} / \mathrm{ml}$ for a gefitinib dose of $225 \mathrm{mg}$ daily (the approved dose in Japan is $250 \mathrm{mg}$ daily) [15], respectively. Togashi et al. reported that the cerebrospinal fluid concentration and penetration rate of erlotinib $(150 \mathrm{mg}$ daily) were significantly higher than those of gefitinib
(250 mg daily) [16]. Because of these factors, erlotinib may be superior to gefitinib for controlling CNS metastasis.

Our study has some limitations. Baseline characteristics varied among the study subjects. This difference may have introduced potential bias, which in turn may have affected the study outcomes. First, more patients had brain metastasis in erlotinib group compared with gefitinib group. In the past report, disruption of the blood-brain barrier (BBB) in the presence of CNS metastasis is likely to lead to locally increased drug concentration [17]. Second, more patients had history of radiotherapy for brain metastasis in erlotinib group than gefitinib group. Zeng et al. reported that whole brain radiotherapy (WBRT) combined with an EGFR-TKI increase the BBB permeability of the EGFR-TKI [18]. Magnuson et al. demonstrated a tendency for upfront stereotactic radiosurgery (SRS) or WBRT followed by an EGFR-TKI 

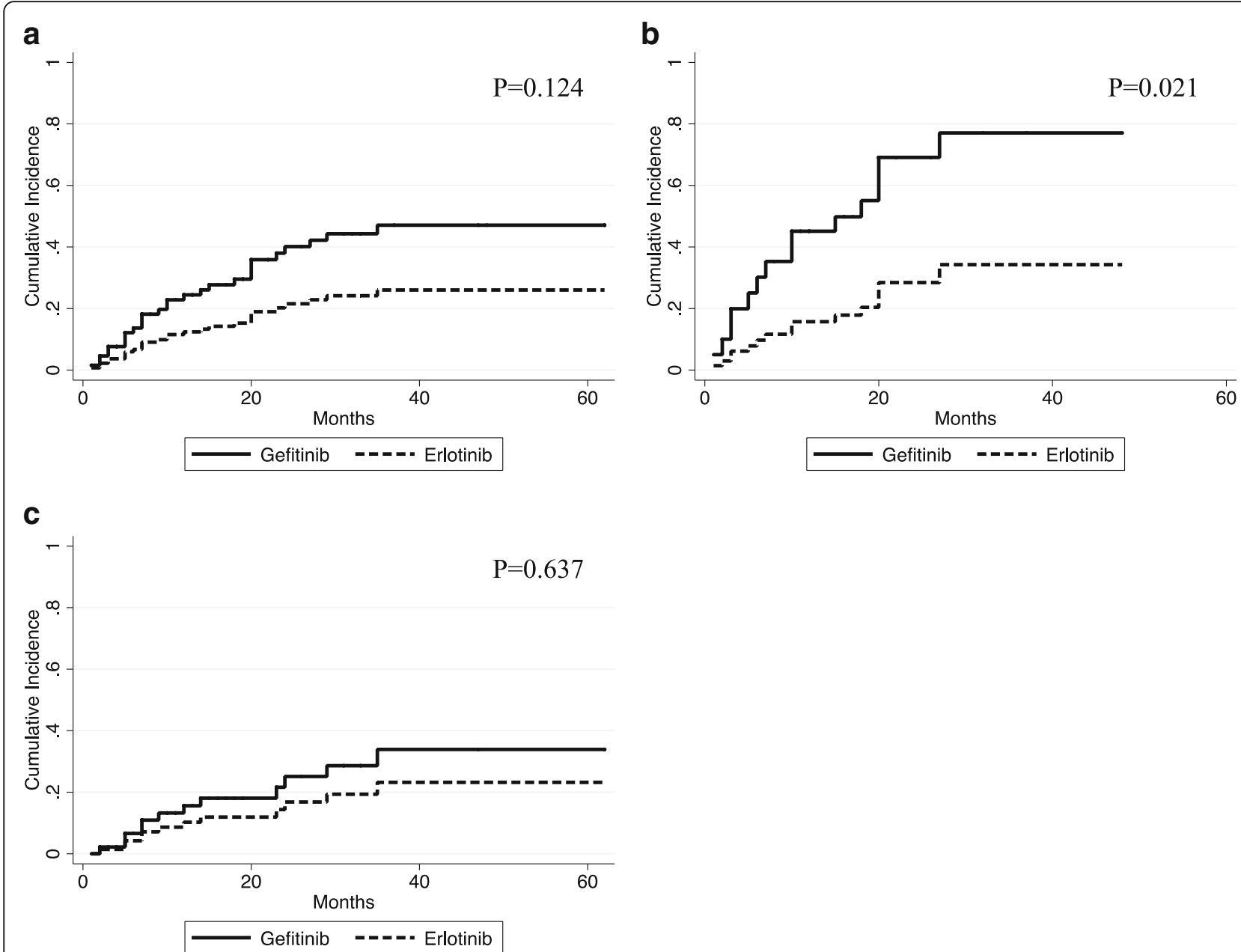

Fig. 2 Cumulative incidence of brain metastasis progression using competing risks regression analysis in patients treated with gefitinib or erlotinib. a All patients in this study. b Patients who had brain metastasis before EGFR-TKI administration. c Patients who had no brain metastasis before EGFR-TKI administration

to decrease intracranial disease progression better than an upfront EGFR-TKI followed by SRS or WBRT [19]. Third, Exon 19 deletion was detected more frequently in erlotinib group than gefitinib group in our study. Lee CK et al. reported that exon 19 deletions

Table 2 Multivariate analysis of the clinical characteristics prognostic of central nervous system progression

\begin{tabular}{llll}
\hline & HR & $95 \% \mathrm{Cl}$ & $P$ value \\
\hline Sex: male vs. female & 0.769 & $0.342-1.729$ & 0.526 \\
Age: $\geqq 70$ vs. $<70$ years & 0.521 & $0.236-1.150$ & 0.107 \\
ECOG PS: $\geqq 2$ vs. $<2$ & 1.013 & $0.284-3.618$ & 0.984 \\
Brain metastasis: yes vs. no & 2.540 & $1.131-5.702$ & 0.024 \\
EGFR-TKI: erlotinib vs. gefitinib & 0.321 & $0.114-0.903$ & 0.031 \\
\hline
\end{tabular}

In the Cox proportional hazard regression model, the variables adjusted for included sex, age, ECOG PS, presence of brain metastasis at the start of EGFRTKI treatment, and the EGFR-TKI agent used

ECOG PS Eastern Cooperative Oncology Group performance status, EGFR-TKI epidermal growth factor receptor tyrosine kinase inhibitor were associated with longer PFS than exon 21 L858R substitution in their meta-analysis [20]. Forth, more of the patients who received gefitinib, compared with the erlotinib, had a poor ECOG PS in this study. While few studies have compared PFS and OS after EGFR-TKI treatment between patients with a good PS and those with a poor PS, Kudoh et al. reported that elderly patients with a poor PS are more likely to develop interstitial lung disease than younger patients with a good PS [21]. These differences of baseline might have had a favorable influence on the patients in the erlotinib group of our study.

On the other hand, more patients had history of chemotherapy prior to EGFR-TKI therapy in erlotinib group than gefitinib group. $\mathrm{Xu} \mathrm{J}$ et al. reported that first-line therapy with EGFR-TKI therapy achieved longer PFS and higher objective response rate (ORR) compared with second line therapy [22]. This factor could have had adverse influence on erlotinib group. 
In addition, due to the retrospective nature of the study brain MRI or CT was not performed routinely but only when clinically indicated, which may have affected the evaluation of the time to CNS progression.

\section{Conclusion}

This retrospective study suggested the value of erlotinib as a more promising treatment for patients with EGFR mutant NSCLC with brain metastasis compared with gefitinib. Further pre-planned and large-scale studies are warranted to confirm these results.

\section{Abbreviations}

BBB: Blood-brain barrier; CNS: Central nervous system; CT: Computed tomography; DCR: Disease control rate; ECOG PS: Eastern Cooperative Oncology Group performance status; EGFR-TKl: Epidermal growth factor receptor tyrosine kinase inhibitor; MRI: Magnetic resonance imaging; NSCLC: Non-small-cell lung cancer; ORR: Objective response rate; OS: Overall survival; PFS: Progression free survival; RR: Response rate; SRS: Stereotactic radiosurgery; WBRT: Whole brain radiotherapy

\section{Acknowledgements}

We thank all of the participants for their participation in the study and for their cooperation during follow-up.

\section{Availability of data and materials}

All relevant data regarding the study conclusion are displayed in the publication. Raw data used during the study are not publicly available because this include some indirect identifying information (age, sex, ECOG PS, TNM classification, the type of EGFR mutation, the initial date of medication, the date of progression disease, and the date of death), but are available from the corresponding author on reasonable request.

\section{Duplicate publication}

We previously reported this study in the IASLC 17th world conference [23].

\section{Authors' contributions}

$\mathrm{NA}, \mathrm{TS}$, and $\mathrm{HO}$ designed the study. NA, TS, KM, YM, YA, MI, YN collected the data. NA and TY analyzed the data. NA prepared the manuscript. All authors read, revised and approved the final manuscript.

\section{Ethics approval and consent to participate}

This retrospective study was conducted in accordance with the ethical standards of the declaration of Helsinki and approved by the Ethics Committee of Yokohama Municipal Citizen's Hospital, Kanagawa, Japan (Approval Number: 17-11-06). The requirement for informed consent was waived due to the retrospective nature of the study.

\section{Consent for publication}

Not applicable.

\section{Competing interests}

The authors declare that they have no competing interests.

\section{Publisher's Note}

Springer Nature remains neutral with regard to jurisdictional claims in published maps and institutional affiliations.

\section{Author details}

'Department of Respiratory Medicine, Yokohama Municipal Citizen's Hospital, 56 Okazawa-cho, Hodogaya-ku, Yokohama-city, Kanagawa 240-8555, Japan. 2Department of Medical Oncology, Yokohama Municipal Citizen's Hospital, 56 Okazawa-cho, Hodogaya-ku, Yokohama-city, Kanagawa 240-8555, Japan. ${ }^{3}$ Department of Biostatistics, Yokohama City University School of Medicine, 3-9 Fukuura, Kanazawa-ku, Yokohama-city, Kanagawa 236-0004, Japan.
Received: 12 September 2017 Accepted: 8 October 2018

Published online: 22 October 2018

\section{References}

1. D'Antonio C, Passaro A, Gori B, et al. Bone and brain metastasis in lung cancer: recent advances in therapeutic strategies. Adv Med Oncol. 2014;6(3): $101--14$

2. Iuchi T, Shingyoji M, Itakura $M$, et al. Frequency of brain metastases in nonsmall cell lung cancer, and their association with epidermal growth factor receptor mutations. Int J Clin Oncol. 2014;20(4):674--9.

3. Mitsudomi T, Morita S, Yatabe Y, et al. Gefitinib versus cisplatin plus docetaxel in patients with non-small-cell lung cancer harbouring mutations of the epidermal growth factor receptor (WJTOG3405): an open label, randomised phase 3 trial. Lancet Oncol. 2010;11(2):121-8.

4. Maemondo M, Inoue A, Kobayashi K, et al. Gefitinib or chemotherapy for non-small-cell lung cancer with mutated EGFR. N Engl J Med. 2010;362(25): 2380--8.

5. Zhou $C$, Wu $Y L$, Chen $G$, et al. Erlotinib versus chemotherapy as first-line treatment for patients with advanced EGFR mutation-positive non-small-cell lung cancer (OPTIMAL, CTONG-0802): a multicentre, open-label, randomised phase 3 study. Lancet Oncol. 2011;12(8):735--42.

6. Rosell R, Carcereny E, Gervais R, et al. Erlotinib versus standard chemotherapy as first-line treatment for European patients with advanced EGFR mutation-positive non-small-cell lung cancer (EURTAC): a multicentre, open-label, randomised phase 3 trial. Lancet Oncol. 2012;13(3):239--46.

7. Namba Y, Kijima T, Yokota S, et al. Gefitinib in patients with brain metastases from non-small-cell lung cancer: review of 15 clinical cases. Clin Lung Cancer. 2004;6(2):123--8.

8. Ceresoli GL, Cappuzzo F, Gregorc V, et al. Gefitinib in patients with brain metastases from non-small-cell lung cancer: a prospective trial. Ann Oncol. 2004;15(7):1042-7.

9. Bai H, Han B. The effectiveness of erlotinib against brain metastases in nonsmall cell lung cancer patients. Am J Clin Oncol. 2013;36(2):110-5.

10. Iuchi T, Shingyoji M, Sakaida T, et al. Phase II trial of gefitinib alone without radiation therapy for Japanese patients with brain metastases from EGFRmutant lung adenocarcinoma. Lung Cancer. 2013;82(2):282--7.

11. Omuro AM, Kris MG, Miller VA, et al. High incidence of disease recurrence in the brain and leptomeninges in patients with nonsmall cell lung carcinoma after response to gefitinib. Cancer. 2005:103:2344-8.

12. Yamamoto N, Goto K, Nishio M. Final overall survival in JO22903, a phase II, open-label study of first-line erlotinib for Japanese patients with EGFR mutation-positive non-small-cell lung cancer. Int J Clin Oncol. 2017;22(1):70-8

13. Urata Y, Katakami N, Morita S, et al. Randomized phase III study comparing gefitinib with erlotinib in patients with previously treated advanced lung adenocarcinoma: WJOG 5108L. J Clin Oncol. 2016;34(27):3248-57.

14. Hidalgo M, Siu LL, Nemunaitis J, et al. Phase I and pharmacologic study of OSI-774, an epidermal growth factor receptor tyrosine kinase inhibitor, in patients with advanced solid malignancies. J Clin Oncol. 2001:19(13):3267--79.

15. Ranson M, Hammond LA, Ferry D, et al. ZD1839, a selective oral epidermal growth factor receptor-tyrosine kinase inhibitor, is well tolerated and active in patients with solid, malignant tumors: results of a phase I trial. J Clin Oncol. 2002;20(9):2240-50.

16. Togashi Y, Masago K, Masuda S, et al. Cerebrospinal fluid concentration of gefitinib and erlotinib in patients with non-small cell lung cancer. Cancer Chemother Pharmaco. 2012;70(3):399-405

17. Jamal-Hanjani $M$, Spicer J. Epidermal growth factor receptor tyrosine kinase inhibitors in the treatment of epidermal growth factor receptor-mutant non-small cell lung cancer metastatic to the brain. Clin Cancer Res. 2012; 18(4):938-44.

18. Zeng YD, Liao H, Qin T, et al. Blood-brain barrier permeability of gefitinib in patients with brain metastases from non-small-cell lung cancer before and during whole brain radiation therapy. Oncotarget. 2015;6(10):8366-76.

19. Magnuson WJ, Lester-Coll NH, Wu AJ, et al. Management of brain metastases in tyrosine kinase inhibitor-naïve epidermal growth factor receptor-mutant non-small-cell lung cancer: a retrospective multi-institutional analysis. J Clin Oncol. 2017. https://doi.org/10.1200/JCO.2016.69.7144.

20. Lee $C K$, Wu YL, Ding PN, et al. Impact of specific epidermal growth factor receptor (EGFR) mutations and clinical characteristics on outcomes after 
treatment with EGFR tyrosine kinase inhibitors versus chemotherapy in EGFR-mutant lung Cancer: a meta-analysis. J Clin Oncol. 2015;33:1958-65.

21. Kudoh $\mathrm{S}$, Kato H, Nishikawa $\mathrm{Y}$, et al. Interstitial lung disease in Japanese patients with lung cancer: a cohort and nested case-control study. Am J Respir Crit Care Med. 2008;177(12):1348-57.

22. Walbert T, Gilbert MR. The role of chemotherapy in the treatment of patients with brain metastases from solid tumors. Int J Clin Oncol. 2009; 14(4):299-306.

23. Aiko N, Shimokawa T, Miyazaki K, et al. Comparison of the efficacy of firstgeneration EGFR-TKIs in brain metastasis. J Thorac Oncol. 2017;12(1):944-5.

Ready to submit your research? Choose BMC and benefit from:

- fast, convenient online submission

- thorough peer review by experienced researchers in your field

- rapid publication on acceptance

- support for research data, including large and complex data types

- gold Open Access which fosters wider collaboration and increased citations

- maximum visibility for your research: over $100 \mathrm{M}$ website views per year

At BMC, research is always in progress.

Learn more biomedcentral.com/submissions 